\title{
Cubic String Boundary Value Problems and Cauchy Biorthogonal Polynomials
}

\author{
M. Bertola ${ }^{\dagger \dagger} 1$, M. Gekhtman ${ }^{a}{ }^{2}$, J. Szmigielski ${ }^{b} 3$ \\ † Centre de recherches mathématiques, Université de Montréal \\ C. P. 6128, succ. centre ville, Montréal, Québec, Canada H3C 3J7 \\ E-mail: bertola@crm.umontreal.ca \\ ¥ Department of Mathematics and Statistics, Concordia University \\ 7141 Sherbrooke W., Montréal, Québec, Canada H4B 1R6 \\ ${ }^{a}$ Department of Mathematics 255 Hurley Hall, Notre Dame, IN 46556-4618, USA \\ E-mail: Michael.Gekhtman.1@nd.edu \\ ${ }^{b}$ Department of Mathematics and Statistics, University of Saskatchewan \\ 106 Wiggins Road, Saskatoon, Saskatchewan, S7N 5E6, Canada
}

E-mail:szmigiel@math.usask.ca

\begin{abstract}
Cauchy Biorthogonal Polynomials appear in the study of special solutions to the dispersive nonlinear partial differential equation called the Degasperis-Procesi (DP) equation, as well as in certain two-matrix random matrix models. Another context in which such biorthogonal polynomials play a role is the cubic string; a third order ODE boundary value problem $-f^{\prime \prime \prime}=z g f$ which is a generalization of the inhomogeneous string problem studied by M.G. Krein. A general class of such boundary value problems going beyond the original cubic string problem associated with the DP equation is discussed under the assumption that the source of inhomogeneity $g$ is a discrete measure. It is shown that by a suitable choice of a generalized Fourier transform associated to these boundary value problems one can establish a Parseval type identity which aligns Cauchy biorthogonal polynomials with certain natural orthogonal systems on $L_{g}^{2}$.
\end{abstract}

\footnotetext{
${ }^{1}$ Work supported in part by the Natural Sciences and Engineering Research Council of Canada (NSERC), Grant. No. 261229-03 and by the Fonds FCAR du Québec No. 88353.

${ }^{2}$ Work supported in part by NSF Grant DMD-0400484.

${ }^{3}$ Work supported in part by the Natural Sciences and Engineering Research Council of Canada (NSERC), Grant. No. 138591-04
} 


\section{An ordinary inhomogeneous string, the Degasperis-Procesi equation and a cubic string}

\subsection{DP equation}

The vibrating string is one of the most fundamental physical phenomena, whose mathematical foundations go back at least to the times of D. Bernoulli with important contributions of J.B. Fourier, B. Riemann, S. Sobolev and M.G. Krein and it appears that the heated discussion of more refined aspects of the problem has not subsided. Of multiple reasons for the special role of the vibrating string two stand out: 1) this is the simplest problem with infinitely many degrees of freedom and as such it inevitably leads to questions of analysis on function spaces, in particular the eigenvalue problems for infinitely large matrices; 2) the vibrating string problem is a prototype of hyperbolic problems and plays an important role in basic mathematical education. In its simplest, one dimensional, form, one studies the partial differential equation

$$
\frac{1}{c^{2}} u_{t t}-u_{x x}=0, \quad 0<x<1,
$$

for the amplitude of the string $u(x, t)$. The coefficient $\frac{1}{c^{2}}$ is proportional to the mass density $\rho$ of the string and in principle it does not have to be homogeneous (constant) in $x$. Thus, in general, $\frac{1}{c^{2}}=\frac{\rho(x)}{T}$, where $T$ represents the tension in the string and $\rho(x)$ is the linear mass density of the string. Then the fundamental modes, $u(x, t)=v(x) \cos (\omega t)$, are given by solving:

$$
-v_{x x}=\frac{\omega^{2}}{T} \rho(x) v, \quad 0<x<1,
$$

subject to some boundary conditions, corresponding physically to how the string is tied. The most common are the Dirichlet boundary conditions, $v(0)=v(1)=0$ (the string is tied on both ends), and the Neumann conditions, $v_{x}(0)=v_{x}(1)=0$ (the end points can slide in the vertical direction only). Once equipped with the boundary conditions, say the Dirichlet conditions, the problem is turned into an eigenvalue problem of the Sturm-Liouville type:

$$
-v_{x x}=z \rho(x) v, \quad v(0)=v(1)=0, \quad 0<x<1
$$

where $z=\frac{\omega^{2}}{T}$. Such an inhomogeneous string problem was studied in an influential work by M.G. Krein [1] in the 1950s as a generalization of Stieltjes' theory of analytic continued fractions [2].

The present paper addresses several aspects of Krein's theory for a third order equation:

$$
-\phi_{x x x}=z \rho \phi, \quad 0<x<1
$$

subject to some boundary conditions specified later. We will refer to this equation as the cubic string. Since any third order problem is non-self adjoint one can seriously doubt any applicability of this type of equation to physical systems. Below we briefly sketch how the cubic string has arisen in a study of certain 
nonlinear partial differential equation modeling weekly dispersive waves. The equation in question is the Degasperis-Procesi (DP) equation [3]:

$$
u_{t}-u_{x x t}+4 u u_{x}=3 u_{x} u_{x x}+u u_{x x x}, \quad(x, t) \in \mathbb{R}^{2},
$$

where $u(x, t)$ is the wave hight at $x$ and time $t$. The DP equation admits a Lax formulation, first proposed in [4], which means that the DP equation follows from the compatibility condition for the the system

$$
\begin{aligned}
\left(\partial_{x}-\partial_{x}^{3}\right) \psi & =z m \psi, \\
\partial_{t} \psi & =\left[z^{-1}\left(1-\partial_{x}^{2}\right)+u_{x}-u \partial_{x}\right] \psi .
\end{aligned}
$$

where $z \in \mathbb{C}$. In general $m$ can be a Radon measure, for example $m$ can be a discrete measure $m=$ $\sum_{i=1}^{n} m_{i} \delta_{x_{i}}$, and, as a result, the equation (1-3) will be assumed to hold in the sense of distributions. It is helpful to bring (1-3a) to its canonical form [5]. To this end one performs a Liouville transformation on (1-3a). This is fully explained in [5] and here we only state the essential results leading to the appearance of the cubic string boundary value problem.

Lemma 1.1. Under the change of variables

$$
\xi=\tanh \frac{x}{2}, \quad \psi(x)=\frac{2 \phi(\xi)}{1-\xi^{2}},
$$

the DP spectral problem (1-3a) is equivalent to the cubic string problem

$$
\begin{aligned}
-\phi_{\xi \xi \xi}(\xi) & =z g(\xi) \phi(\xi) \quad \text { for } \xi \in(-1,1), \\
\phi(-1)=\phi_{\xi}(-1) & =0, \\
\phi(1) & =0,
\end{aligned}
$$

where

$$
\left(\frac{1-\xi^{2}}{2}\right)^{3} g(\xi)=m(x)
$$

In the discrete case, when $m(x)=2 \sum_{1}^{n} m_{i} \delta_{x_{i}}$, equation (1-6) should be interpreted as

$$
g(\xi)=\sum_{i=1}^{n} g_{i} \delta_{\xi_{i}}, \quad \text { where } \quad \xi_{i}=\tanh \frac{x_{i}}{2}, \quad g_{i}=\frac{8 m_{i}}{\left(1-\xi_{i}^{2}\right)^{2}} .
$$

To solve the original DP equation requires solving the spectral and inverse spectral problem as explained in detail in [5] for the case of the finite discrete measure $m$. Our goal in the remainder of the paper is to present a few essential aspects of the spectral problem associated to the cubic string for a variety of boundary conditions with the view towards explaining the role of a novel class of Cauchy biorthogonal polynomials introduced in $[6,7]$. These polynomials can be defined for arbitrary positive measures $d \alpha, d \beta$ supported on the positive part of the real axis $\mathbb{R}_{+}$provided that all (Cauchy) bimoments $I_{i j}=\iint \frac{x^{i} y^{j}}{x+y} d \alpha(x) d \beta(y)$ are finite. One then defines polynomials $q_{j}(y), p_{j}(x)$ of degree $j=0,1, \ldots$ satisfying the biorthogonality condition $\iint \frac{p_{j}(x) q_{k}(y)}{x+y} d \alpha(x) d \beta(y)=\delta_{j k}$. We refer to these as Cauchy biorthogonal polynomials. 


\section{Discrete cubic strings}

We slightly generalize the cubic string discussed in the previous section in connection with the DP equation. We recall that in an ordinary string problem different boundary conditions correspond to different ways of tying down the ends of the string. For us, different boundary conditions will eventually lead to different spectral measures with respect to which we will define biorthogonal polynomials. However, in all cases discussed by us the spectrum is positive and simple, as one would expect from any vibrating system.

Definition 2.1. The cubic string boundary value problems:

$$
\begin{aligned}
& -\phi_{\xi \xi \xi}(\xi)=z g(\xi) \phi(\xi), \quad 0<\xi<1, \quad \phi(0)=\phi_{\xi}(0)=0, \\
& \text { Type } 0(\text { DP case }): \phi(1)=0, \quad \text { Type } 1: \phi_{\xi}(1)=0 \quad \text { Type } 2: \phi_{\xi \xi}(1)=0
\end{aligned}
$$

Remark 2.1. For simplicity we have adjusted the length of the string; it is now 1 rather than 2.

We are only interested in the case where the mass distribution consists of a finite collection of pointmasses:

$$
g(\xi)=\sum_{i=1}^{n} g_{i} \delta_{\xi_{i}}, \quad \text { where } \quad 0<g_{i}, \quad 0<\xi_{1}<\xi_{2}<\cdots<\xi_{n}<1 .
$$

We will consider all three boundary value problems mentioned above with this mass distribution, as well as one degenerate case in which the last mass is placed at 1 (i.e. $\xi_{n}=1$ : in the latter case we take the right hand limit to compute the derivatives of $f$ at 1 . Moreover, for that case, we consider only the BVP of type 2.)

We will collectively refer to all these cases as the discrete cubic string problem.

We will also use an accompanying initial value problem,

$$
\phi(0)=\phi_{\xi}(0)=0, \phi_{\xi \xi}(0)=1 .
$$

The boundary value problems in Definition 2.1 are not self-adjoint and the adjoint boundary value problems play an important role.

Definition 2.2. The adjoint cubic string boundary value problems:

$$
\phi_{\xi \xi \xi}^{*}(\xi)=z g(\xi) \phi^{*}(\xi), \quad 0<\xi<1,
$$

Type 0: $\phi^{*}(1)=\phi_{\xi}^{*}(1)=0, \phi^{*}(0)=0$, Type 1: $\phi^{*}(1)=\phi_{\xi \xi}^{*}(1)=0, \phi^{*}(0)=0$, Type $2: \phi_{\xi}^{*}(1)=\phi_{\xi \xi}^{*}(1)=0, \phi^{*}(0)=0$.

The corresponding initial value problems are: 
Definition 2.3. The adjoint cubic string initial value problems:

$$
\phi_{\xi \xi \xi}^{*}(\xi)=z g(\xi) \phi^{*}(\xi) \quad 0<\xi<1
$$

with nonzero initial values: Type 0: $\phi_{\xi \xi}^{*}(1)=1$, Type 1: $\phi_{\xi}^{*}(1)=1$, Type 2: $\phi^{*}(1)=1$.

Since all three boundary value problems for $\phi$ satisfy the same initial value problem we will use one letter, namely $\phi$, to denote the solution. However, we will attach an index $a=0,1,2$ to $\phi^{*}$ to indicate the type of the BVP; for example, $\phi_{0}^{*}$ will refer to the BVP/IVP of Type 0 etc.

In the process of integration by parts of expressions like $\int_{0}^{1} f_{\xi \xi \xi}(\xi) h(\xi) d \xi$ one identifies the relevant bilinear symmetric form:

Definition 2.4. Given any twice differentiable $f, h$ the bilinear concomitant is defined as the bilinear form:

$$
B(f, h)(\xi)=f_{\xi \xi} h-f_{\xi} h_{\xi}+f h_{\xi \xi} .
$$

This bilinear symmetric form induces a bilinear symmetric form (denoted also by B) on triples $F^{T}:=$ $\left(f, f_{\xi}, f_{\xi \xi}\right)$, namely

$$
B(F, H)=F^{T} J H, \quad J:=\left(\begin{array}{ccc}
0 & 0 & 1 \\
0 & -1 & 0 \\
1 & 0 & 0
\end{array}\right) .
$$

Furthermore, we also define a natural $L^{2}$ space associated with $g$, denoted $L^{2}[0,1]_{g}$, equipped with the inner product: $(f, h)_{g}=\int_{0}^{1} f(\xi) h(\xi) g(\xi) d \xi$. Whenever $f$ or $g$ depend on the spectral variables $z$ and $\lambda$, we write $(f(z), g(\lambda))_{g}$ to display this dependence.

Since all initial value problems 2-3, 2.3 can be solved for arbitrary $z \in \mathbb{C}, \phi$ and $\phi_{a}^{*}$ are functions of the spectral parameter $z$. The following theorem establishes a relation between these two functions in terms of their concomitant and the relevant boundary value problem. The customary notation: $\left.f(\xi)\right|_{0} ^{1}=$ $f(1)-f(0)$ is used in the statement below.

Lemma 2.1. Suppose $\phi(\xi ; z)$ and $\phi_{a}^{*}(\xi ; \lambda)$ are solutions to the IVPs 2-3, 2.3 with spectral parameters $z$, $\lambda$ respectively. Then

1. Type 0: the spectrum is determined by the zeros of $\phi(1, z)=0$ and

$$
-\left.B\left(\phi(\xi ; z), \phi_{0}^{*}(\xi, \lambda)\right)\right|_{0} ^{1}=\phi_{0}^{*}(0 ; \lambda)-\phi(1 ; z)=(z-\lambda)\left(\phi(z), \phi_{0}^{*}(\lambda)\right)_{g}
$$

2. Type 1: the spectrum is determined by the zeros of $\phi_{\xi}(1, z)=0$ and

$$
-\left.B\left(\phi(\xi ; z), \phi_{1}^{*}(\xi, \lambda)\right)\right|_{0} ^{1}=\phi_{1}^{*}(0 ; \lambda)+\phi_{\xi}(1 ; z)=(z-\lambda)\left(\phi(z), \phi_{1}^{*}(\lambda)\right)_{g} .
$$


3. Type 2: the spectrum is determined by the zeros of $\phi_{\xi \xi}(1, z)=0$ and

$$
-\left.B\left(\phi(\xi ; z), \phi_{2}^{*}(\xi, \lambda)\right)\right|_{0} ^{1}=\phi_{2}^{*}(0 ; \lambda)-\phi_{\xi \xi}(1 ; z)=(z-\lambda)\left(\phi(z), \phi_{2}^{*}(\lambda)\right)_{g} .
$$

In addition,

$$
-\left.B(\phi(\xi ; z), \phi(\xi ; \lambda))\right|_{0} ^{1}=(z+\lambda)(\phi(z), \phi(\lambda))_{g} .
$$

Proof. Indeed (2-3) and two integrations by parts imply that

$$
\begin{aligned}
-\int_{0}^{1} \phi_{\xi \xi \xi}(\xi ; z) \phi_{a}^{*}(\xi ; \lambda) d \xi= & -\left.B\left(\phi, \phi_{a}^{*}\right)\right|_{0} ^{1}+\int_{0}^{1} \phi(\xi ; z) \phi_{a, \xi \xi \xi}^{*}(\xi ; \lambda) d \xi= \\
& z \int_{0}^{1} \phi(\xi ; z) \phi_{a}^{*}(\xi ; \lambda) g(\xi) d \xi .
\end{aligned}
$$

Consequently, using equation (2-5) we obtain:

$$
-\left.B\left(\phi, \phi_{a}^{*}\right)\right|_{0} ^{1}=(z-\lambda) \int_{0}^{1} \phi(\xi ; z) \phi_{a}^{*}(\xi ; \lambda) g(\xi) d \xi
$$

which in view of the initial conditions implies the claim. A similar computation works for the second identity.

By specializing $\lambda=z$ in the lemma above one readily obtains:

Corollary 2.1. $\phi$ and $\phi_{a}^{*}$ satisfy the following relations:

1. Case 0: $\phi_{0}^{*}(0 ; z)=\phi(1 ; z)$. Case $1: \phi_{1}^{*}(0 ; z)=-\phi_{\xi}(1 ; z)$, Case $2: \phi_{2}^{*}(0 ; z)=\phi_{\xi \xi}(1 ; z)$.

2. Case 0: $-\phi_{z}(1 ; z)=\left(\phi(z), \phi_{0}^{*}(z)\right)_{g}$. Case 1: $\phi_{\xi z}(1 ; z)=\left(\phi(z), \phi_{1}^{*}(z)\right)_{g}$. Case 2: $-\phi_{\xi \xi z}(1 ; z)=$ $\left(\phi(z), \phi_{2}^{*}(z)\right)_{g}$.

We give below a complete characterization of the spectra and the corresponding eigenfunctions for all three BVPs. We also select certain combinations of BVPs which reveal the origin of the relevance of the Cauchy kernel $\frac{1}{x+y}$ to the spectral theory of the cubic string.

Theorem 2.1. Consider a cubic string with a finite measure $g$ as in (2-2).

1. Let $z_{a, j}$ denote the eigenvalues of the BVP of Type $a=0,1,2$. In each of the three cases, the spectrum is positive and simple.

2. For any pair of BVPs of Type 0,1,2 the spectra are interlaced in the following order:

$$
z_{2, j}<z_{1, j}<z_{0, j}, \quad j=1, \ldots n
$$

3. For each $a=0,1,2$ the eigenfunctions $\phi\left(\xi ; z_{a, j}\right) \equiv \phi_{a, j}(\xi)$ are linearly independent. 
4. For the following combinations of BVPs, $\left(\phi_{a, i}, \phi_{b, j}\right)_{g}$ factorizes:

(a) Type 00:

$$
\left(\phi_{0, i}, \phi_{0, j}\right)_{g}=\frac{\phi_{0, i, \xi}(1) \phi_{0, j, \xi}(1)}{z_{0, i}+z_{0, j}}
$$

(b) Type 01

$$
\left(\phi_{0, i}, \phi_{1, j}\right)_{g}=-\frac{\phi_{0, i, \xi \xi}(1) \phi_{1, j}(1)}{z_{0, i}+z_{1, j}}
$$

(c) Type 12

$$
\left(\phi_{1, i}, \phi_{2, j}\right)_{g}=-\frac{\phi_{1, i, \xi \xi}(1) \phi_{2, j}(1)}{z_{1, i}+z_{2, j}}
$$

(d) Type 22

$$
\left(\phi_{2, i}, \phi_{2, j}\right)_{g}=\frac{\phi_{2, i, \xi}(1) \phi_{2, j, \xi}(1)}{z_{2, i}+z_{2, j}}
$$

Proof. It is easy to check (see Section 4.1 in [5]) that

$$
\left(\begin{array}{c}
\phi(1 ; z) \\
\phi_{\xi}(1 ; z) \\
\phi_{\xi \xi}(1 ; z)
\end{array}\right)=L_{n} G_{n}(z) L_{n-1} G_{n-1}(z) \cdots L_{1} G_{1}(z) L_{0}\left(\begin{array}{c}
0 \\
0 \\
1
\end{array}\right) .
$$

where

$$
\begin{gathered}
G_{k}(z)=\left(\begin{array}{ccc}
1 & 0 & 0 \\
0 & 1 & 0 \\
-z g_{k} & 0 & 1
\end{array}\right) \\
L_{k}=\left(\begin{array}{ccc}
1 & l_{k} & l_{k}^{2} / 2 \\
0 & 1 & l_{k} \\
0 & 0 & 1
\end{array}\right),
\end{gathered}
$$

and

$$
l_{k}=\xi_{k+1}-\xi_{k}, \quad \xi_{0}=0, \quad \xi_{n+1}=1 .
$$

We prove the statement about the spectra by using the results obtained in [5]. By Theorem 3.5 in [5] $\phi(1 ; z)$ has $n$ distinct positive zeros and so do $\phi_{\xi}(1 ; z)$ and $\phi_{\xi \xi}(1 ; z)$ (denoted there $\phi_{y}, \phi_{y y}$ ). The second statement follows if one observes that $\frac{\phi_{\xi}(1 ; z)}{\phi_{z}(1 ; z)}$ and $\frac{\phi_{\xi \xi}(1 ; z)}{\phi_{z}(1 ; z)}$ are strictly positive on the spectrum of Type 0 being the residues of $\frac{\phi_{\xi}(1 ; z)}{\phi_{(1 ; z)}}, \frac{\phi_{\xi \xi}(1 ; z)}{\phi_{(1 ; z)}}$ respectively (Theorem 3.5 and Theorem 3.15 in [5]). It follows then that both $\phi_{\xi}(1 ; z)$ and $\phi_{\xi \xi}(1 ; z)$ change signs $n$ times, hence all three spectra are simple. Furthermore, $\phi_{\xi}(1 ; z)>0, \phi_{\xi \xi}(1 ; z)>0$ for $z \leq 0$, so the zeros of $\phi_{\xi}(1 ; z)$ and $\phi_{\xi \xi}(1 ; z)$ are strictly positive. Since $\phi_{\xi}(1 ; z)$ and $\phi_{\xi \xi}(1 ; z)$ change sign on every interval between two consecutive zeros of $\phi(1 ; z)$ the spectra of Type 0 and 1 , as well as 0 and 2, interlace. To see that the spectrum of Type 1 interlaces with the spectrum of Type 2 we proceed as follows. By (2-11), after evaluating at $z=z_{2, i}, \lambda=-z_{2, i}$, we obtain

$$
\phi_{\xi}\left(1 ; z_{2, i}\right) \phi_{\xi}\left(1 ;-z_{2, i}\right)=\phi_{\xi \xi}\left(1 ;-z_{2, i}\right) \phi\left(1 ; z_{2, i}\right)
$$


which gives

$$
\phi_{\xi}\left(1 ; z_{2, i}\right)=\frac{\phi_{\xi \xi}\left(1 ;-z_{2, i}\right)}{\phi_{\xi}\left(1 ;-z_{2, i}\right)} \phi\left(1 ; z_{2, i}\right)
$$

For $z>0, \operatorname{sgn}\left(\phi_{\xi}(1 ;-z)\right)=\operatorname{sgn}\left(\phi_{\xi \xi}(1 ;-z)\right)=+1$ because both are strictly positive there. Since the zeros of $\phi(1 ; z)$ interlace with the zeros of $\phi_{\xi \xi}(1 ; z), \operatorname{sgn}\left(\phi\left(1 ; z_{2, i}\right)\right)$ alternates, which in turn implies that $\operatorname{sgn}\left(\phi_{\xi}\left(1 ; z_{2, i}\right)\right)$ alternates as well. Thus the zeros of $\phi_{\xi}(1 ; z)$ interlace with the zeros of $\phi_{\xi \xi}(1 ; z)$.

The relative position of the spectra of the three types is best inferred from the fact that on the first eigenvalue $z_{0,1}$ of Type $0, \phi_{\xi}\left(1 ; z_{0,1}\right)$ and $\phi_{\xi \xi}\left(1 ; z_{0,1}\right)$ are both negative since $\frac{\phi_{\xi}(1, z)}{\phi_{z}(1, z)}$ and $\frac{\phi_{\xi \xi}(1, z)}{\phi_{z}(1, z)}$ are strictly positive on the spectrum of Type 0 . Thus the spectra of Type 1 and 2 are shifted to the left relative to the spectrum of Type 0 . In particular, $\phi\left(1, z_{2,1}\right)>0$ and so is $\phi_{\xi}\left(1, z_{2,1}\right)$ by $(2-18)$. Thus, at least the first zero of $\phi_{\xi \xi}$ occurs to the left of the zeros of $\phi_{\xi}$ and $\phi$. So $z_{2,1}<z_{1,1}<z_{0,1}$. Suppose this holds for the $(j-1)$ st eigenvalues. Then we know that both $z_{0, j-1}<z_{1, j}<z_{0, j}$ and $z_{0, j-1}<z_{2, j}<z_{0, j}$. If $z_{1, j}<z_{2, j}$ then $z_{2, j-1}<z_{1, j-1}<z_{1, j}<z_{2, j}$, thus contradicting that the spectra of type 1 and 2 interlace.

As for the linear independence we observe that the cubic string boundary value problem 2.1 can be written as an integral equation:

$$
\phi(\xi ; z)=z \int_{0}^{1} G(\xi, \tau) \phi(\tau ; z) g(\tau) d \tau,
$$

where $G(\xi, \tau)$ is the Green function satisfying the boundary conditions of 2.1. Then the linear independence of eigenfunctions corresponding to distinct eigenvalues follows from the general result about the eigenfunctions of a linear operator.

Finally, the statements about the eigenfunctions follow immediately from equation (2-11) after setting $z=z_{a, i}, \lambda=z_{b, j}$.

We now briefly analyze the degenerate case with the mass $m_{n}$ at the end point $x_{n}=1$. We do it only to illustrate that even though the spectrum degenerates in this case the overall conclusions hold.

Theorem 2.2. Let us consider a cubic string with a finite measure $g$ as in (2-2) with $x_{n}=1$. Then

1. the spectra of Type 0,1 , and 2 are positive, simple and their cardinalities are $n-1$ for Type 0 and Type 1 and $n$ for Type 2,

2. the spectra interlace

$$
0<z_{2,1}<z_{1,1}<z_{0,1}<\cdots<z_{2, n-1}<z_{1, n-1}<z_{0, n-1}<z_{2, n}
$$

3. the eigenfunctions $\phi\left(\xi ; z_{2, j}\right):=\phi_{2, j}(\xi)$ satisfy

$$
\left(\phi_{2, i}, \phi_{2, j}\right)_{g}=\frac{\phi_{2, i, \xi}(1) \phi_{2, j, \xi}(1)}{z_{2, i}+z_{2, j}},
$$

and they are linearly independent. 
Remark 2.2. We notice that $\operatorname{deg} \phi(1 ; z)=\operatorname{deg} \phi_{\xi}(1 ; z)=n-1$ while $\operatorname{deg} \phi_{\xi \xi}(1 ; z)=n$. This is in contrast to the previous cases with all positions $x_{1}, \ldots, x_{n}$ inside the interval $[0,1]$ for which all polynomials have the same degree $n$.

Proof. The spectrum of Type 2 is clearly given by the zeros of $\phi_{\xi \xi}(1 ; z)$. Let us first consider the case when $m_{n}$ is placed slightly to the left of the point 1 . Thus, initially, $l_{n}>0$ (see (2-16)). By Theorem 2.1 $\phi(1 ; z)$ has $n$ distinct positive zeros and so does $\phi_{\xi \xi}$ and they interlace. We subsequently take the limit $l_{n} \rightarrow 0$ in the above formulas. We will use the same letters for the limits to ease the notation. By simple perturbation argument, $z_{n, 0} \rightarrow \infty$. Since $z=0$ is not in the spectrum, $z_{2,1}$ has to stay away from 0 . This shows that the spectrum is positive. Furthermore, in the limit $z_{0,1}, \ldots, z_{0, n-1}$ approach simple zeros of the BVP of type 0 for $n-1$ masses. Indeed, using (2-16) with $l_{n}=0$ there, we obtain:

$\phi(1+0 ; z)=\phi(1-0 ; z), \quad \phi_{\xi}(1+0 ; z)=\phi_{\xi}(1-0 ; z), \quad \phi_{\xi \xi}(1+0 ; z)=-z m_{n} \phi(1-0 ; z)+\phi_{\xi \xi}(1-0 ; z)$,

where $1 \pm 0$ refers to the right hand, the left hand limit at 1 respectively. To see that the spectrum is simple we observe that if in the limit two successive eigenvalues coalesce, namely $z_{2, i}=z_{2, i+1}$, then necessarily $z_{2, i}=z_{0, i}$ because of the interlacing property. However now equation (2-21) implies that $\phi\left(1-0 ; z_{0, i}\right)=\phi_{\xi \xi}\left(1-0 ; z_{0, i}\right)=0$ which contradicts Theorem 2.1 for the BVP of Type 0 for $n-1$ masses. Thus, the zeros of $\phi(1, z)$ and $\phi_{\xi \xi}(1+0, z)$ interlace and we have

$$
0<z_{2,1}<z_{0,1}<\cdots<z_{2, n-1}<z_{0, n-1}<z_{2, n} .
$$

Likewise, for the spectrum of Type $1, z_{n, 1} \rightarrow \infty$ and the remaining roots interlace according to the pattern valid for $n-1$ masses.

To prove the statement about the eigenfunctions we use (2-11) and after setting $z=z_{i}, \lambda=z_{j}$ in that formula we obtain the required identity. The linear independence is proven by the same type of argument as in the proof of Theorem 2.1.

We immediately have several results about the adjoint cubic string 2.2.

Corollary 2.2. Given a discrete finite measure $g$

1. for each of the three types of the BVPs the adjoint cubic string (Definition 2.2) and the cubic string (Definition 2.1) have identical spectra.

2. the families of eigenfunctions $\left\{\phi_{a, j}\right\}$ and $\left\{\phi_{a, j}^{*}\right\}$ are biorthogonal, that is:

$$
\left(\phi_{a, i}, \phi_{a, j}^{*}\right)_{g}=0 \quad \text { whenever } \quad i \neq j .
$$


3. For $i=j$,

$$
\left(\phi_{a, i}, \phi_{a, i}^{*}\right)_{g}= \begin{cases}-\phi_{z}\left(1 ; z_{0, i}\right) \neq 0, & a=0 \\ \phi_{\xi z}\left(1 ; z_{1, i}\right) \neq 0, & a=1 \\ -\phi_{\xi \xi z}\left(1 ; z_{2, i}\right) \neq 0, & a=2\end{cases}
$$

holds.

Proof. The first equality in Corollary 2.1 implies that the spectra of the cubic string and its adjoint are identical. The biorthogonality follows immediately from equations (2-8), (2-9) and (2-10). For $i=j$ we use Corollary 2.1. Finally, since the spectrum is simple the required derivatives with respect to $z$ are nonzero.

We conclude this section with the definition and some fundamental properties of two important functions which play a significant role in the theory (Section 6 in [6]).

Definition 2.5. The following functions are called Weyl functions for their respective BVPs:

$$
\begin{aligned}
& \text { Type } 0: W(z):=\frac{\phi_{\xi}(1 ; z)}{\phi(1 ; z)}, \quad Z(z)=\frac{\phi_{\xi \xi}(1 ; z)}{\phi(1 ; z)} ; \quad \text { Type } 1: W(z):=-\frac{\phi(1 ; z)}{\phi_{\xi}(1 ; z)}, \quad Z(z)=\frac{\phi_{\xi \xi}(1 ; z)}{\phi_{\xi}(1 ; z)} ; \\
& \text { Type 2 : } W(z):=-\frac{\phi_{\xi}(1 ; z)}{\phi_{\xi \xi}(1 ; z)}, \quad Z(z)=-\frac{\phi(1 ; z)}{\phi_{\xi \xi}(1 ; z)} .
\end{aligned}
$$

Remark 2.3. The definition of the Weyl functions for the BVP of type 2 in the degenerate case is identical to the one given above for the BVP of type 2.

The Weyl functions $W$ and $Z$ are not independent, they are related by an identity which was originally formulated for the DP peakons in [5]. As an example we formulate such an identity for the BVP of type 2 (both the degenerate as well as the nondegenerate case).

Lemma 2.2. Consider the BVP of type 2. Then the corresponding Weyl functions satisfy:

$$
W(z) W(-z)+Z(z)+Z(-z)=0
$$

Proof. By formula (2-11)

$$
\left.B(\phi(\xi ; z), \phi(\xi ;-z))\right|_{0} ^{1}=0,
$$

which, when written out explicitly, gives the identity:

$$
\phi_{\xi \xi}(1 ; z) \phi(1 ;-z)-\phi_{\xi}(1 ; z) \phi_{\xi}(1 ;-z)+\phi_{\xi \xi}(1 ;-z) \phi(1 ; z)=0 .
$$

Upon dividing the last equation by $\phi_{\xi \xi}(1 ; z) \phi_{\xi \xi}(1 ;-z)$ we obtain the claim.

We state now the spectral representation theorem for $W(z)$ and $Z(z)$ for the BVP of Type 2 in the degenerate case, the remaining cases being merely variations of this, most transparent case. One recognizes again the presence of the Cauchy kernel $\frac{1}{x+y}$ in the spectral representation of $Z(z)$. 
Theorem 2.3. Consider the BVP of type 2 (degenerate case). Then the Weyl functions $W$ and $Z$ have the following (Stieltjes) integral representations:

$$
W(z)=\int \frac{1}{z-y} d \beta(y), \quad Z(z)=\int \frac{1}{(z-y)(x+y)} d \beta(y) d \beta(x),
$$

where $d \beta=\sum_{i=1}^{n} b_{i} \delta_{z_{2, i}}$ and $b_{i}=\frac{-\phi_{\xi}\left(1 ; z_{2, i}\right)}{\phi_{\xi \xi z}\left(1 ; z_{2, i}\right)}>0$.

Proof. Since $\phi_{\xi}(1 ; z), \phi_{\xi \xi}(1 ; z)$ have simple, interlacing zeros, and $\operatorname{deg} \phi_{\xi}(1 ; z)=n-1$ while $\operatorname{deg} \phi_{\xi \xi}(1 ; z)=$ $n, W(z)$ admits a partial fraction decomposition with simple factors:

$$
W(z)=\sum_{i=1}^{n} \frac{b_{i}}{z-z_{2, i}},
$$

where, by the residue calculus, $b_{i}=\frac{-\phi_{\xi}\left(1 ; z_{2, i}\right)}{\phi_{\xi \xi z}\left(1 ; z_{2, i}\right)}$. Moreover the $b_{i}$ s are all of the same sign because the zeros of $\phi_{\xi}(1 ; z)$ and $\phi_{\xi \xi}(1 ; z)$ interlace and, consequently, it suffices to check the sign of $\frac{-\phi_{\xi}\left(1 ; z_{2, i}\right)}{\phi_{\xi \xi z}\left(1 ; z_{2, i}\right)}$ at the first zero $z_{2,1}$. By Theorem $2.1 \operatorname{sgn}\left(\phi_{\xi}\left(1 ; z_{2,1}\right)=1\right.$, and thus $b_{1}>0$ because on the first zero $\phi_{\xi \xi z}$ must be negative. Consequently, all $b_{i}>0$.

Likewise, $Z(z)$ admits a partial fraction decomposition:

$$
Z(z)=\sum_{i=1}^{n} \frac{c_{i}}{z-z_{2, i}}
$$

and again, it follows from the second item in Theorem 2.1 that $c_{i}>0$. Finally, by residue calculus, it follows from Lemma 2.2 that

$$
c_{i}=\sum_{j=1}^{n} \frac{b_{i} b_{j}}{z_{2, i}+z_{2, j}},
$$

which proves the integral representation for $Z(z)$.

\section{Generalized Fourier transform and biorthogonality}

Since $\phi_{a, i}$ are linearly independent we can decompose any $f \in L_{g}^{2}[0,1]$ in the basis of $\left\{\phi_{a, i}\right\}$ and use the dual family $\left\{\phi_{a, i}^{*}\right\}$ to compute the coefficients in the expansion:

$$
f=\sum_{i} C_{a, i} \phi_{a, i}, \quad C_{a, i}=\frac{\left(\phi_{a, i}^{*}, f\right)_{g}}{\left(\phi_{a, i}^{*}, \phi_{a, i}\right)_{g}} .
$$

For each pair $a, b$ for which $\left(\phi_{a, i}, \phi_{b, j}\right)_{g}$ factorizes (item 3 in Theorem 2.1) we define two (finite dimensional) Hilbert spaces $H_{\alpha}:=L^{2}(\mathbb{R}, d \alpha)$ and $H_{\beta}:=L^{2}(\mathbb{R}, d \beta)$ where the measures $d \alpha$ and $d \beta$ are chosen as follows: 1) using Theorem 2.1 split the numerator of $\left.\left(\phi_{a, i}, \phi_{b, j}\right)_{g}, 2\right)$ perform the partial fraction decomposition of the Weyl functions with numerators matching the factors in $\left.\left(\phi_{a, i}, \phi_{b, j}\right)_{g}, 3\right)$ represent the partial fraction decompositions as Stieltjes' transforms of the respective measures. 
Example 3.1. For Type 00, item 3 in Theorem 2.1 states: $\left(\phi_{0, i}, \phi_{0, j}\right)_{g}=\frac{\phi_{0, i, \xi}(1) \phi_{0, j, \xi}(1)}{z_{0, i}+z_{0, j}}$. Hence the numerator splits into $\phi_{0, i, \xi}(1)$ and $\phi_{0, j, \xi}(1)$ and the corresponding Weyl function will be $W(z)=\frac{\phi_{\xi}(1 ; z)}{\phi(1 ; z)}$ (taken twice) with partial fraction decomposition $W(z)=\int \frac{1}{z-x} d \alpha(x)$ where $\mathrm{d} \alpha=d \beta=\sum_{i} \frac{\phi_{0, i . \xi}}{\phi_{z}\left(1 ; z_{0, i}\right)} \delta_{z_{0, i}}$ For Type 01, item 3 in Theorem 2.1 states $\left(\phi_{0, i}, \phi_{1, j}\right)_{g}=-\frac{\phi_{0, i, \xi \xi}(1) \phi_{1, j}(1)}{z_{0, i}+z_{1, j}}$ and the numerator splits into $\phi_{0, i, \xi \xi}(1)$ and $\phi_{1, j}(1)$ which, in turn, match with $Z(z)$ for Type 1 and $W(z)$ for Type 2 in the Definition 2.5. The resulting partial fractions decompositions $Z(z)=\int \frac{1}{z-x} d \alpha(x), W(z)=\int \frac{1}{z-y} d \beta(y)$ imply $\mathrm{d} \alpha=\sum_{i} \frac{\phi_{0, i, \xi \xi}}{\phi_{z}\left(1 ; z_{0, i}\right)} \delta_{z_{0, i}}, \mathrm{~d} \beta=-\sum_{i} \frac{\phi_{1, i}}{\phi_{\xi z}\left(1 ; z_{1, i}\right)} \delta_{z_{1, i}}$.

In summary, for every pair $\{a, b\}$ of BVPs for the cubic string we associate two Hilbert spaces $H_{\alpha}$ and $H_{\beta}$ with the pairing $H_{\alpha} \times H_{\beta} \rightarrow \mathbb{C}$ :

Definition 3.1.

$$
\langle p \mid q\rangle=\int \frac{p(x) q(y)}{x+y} d \alpha(x) \beta(y), \quad p \in H_{\alpha}, \quad q \in H_{\beta} .
$$

We now introduce a family of generalized Fourier transforms adapted to each of the three types of BVPs

Definition 3.2. Given $f \in L_{g}^{2}[0,1]$ and $a=0,1,2$

$$
\hat{f}_{a}(z):=(-1)^{a} \int_{0}^{1} \phi_{a}^{*}(\xi ; z) f(\xi) g(\xi) d \xi .
$$

Remark 3.1. Observe that $\hat{f}_{a}(z)=\left((-1)^{a} \phi_{a}^{*}(z), f\right)_{g}$, and, in particular, $\hat{f}_{a}\left(z_{a, i}\right)=\left((-1)^{a} \phi_{a, i}^{*}, f\right)_{g}$ whenever $z$ equals to one of the points of the spectrum of (2-1).

Remark 3.2. A map of this type was introduced in the context of the inhomogeneous string problem by I.S. Kac and M.G. Krein in [8] as a generalization of the Fourier transform. A similar map known as a distorted Fourier transform is commonly used in Quantum Mechanics.

The main property of this map is captured in the following theorem.

Theorem 3.1. For every pair a, b for which $\left(\phi_{a, i}, \phi_{b, j}\right)_{g}$ factorizes (item 3 in Theorem 2.1) the generalized Fourier transforms satisfy Parseval's identity, that is, for every $f, h \in L_{g}^{2}[0,1]$

$$
(f, h)_{g}=\left\langle\hat{f}_{a} \mid \hat{h}_{b}\right\rangle=\left\langle\hat{h}_{a} \mid \hat{f}_{b}\right\rangle .
$$

Proof. Let us fix $a, b$ for which $\left(\phi_{a, i}, \phi_{b, j}\right)_{g}$ factorizes. Consider two functions $f, h \in L_{g}^{2}[0,1]$. Writing their expansions in the bases $\left\{\phi_{a, i}\right\},\left\{\phi_{b, i}\right\}$ respectively, we obtain

$$
f=\sum_{i} \frac{\left(\phi_{a, i}^{*}, f\right)_{g}}{\left(\phi_{a, i}^{*}, \phi_{a, i}\right)_{g}} \phi_{a, i}, \quad h=\sum_{j} \frac{\left(\phi_{b, j}^{*}, h\right)_{g}}{\left(\phi_{b, j}^{*}, \phi_{b, j}\right)_{g}} \phi_{b, j} .
$$


Hence their inner product reads:

$$
(f, h)_{g}=\sum_{i, j} \frac{\left(\phi_{a, i}^{*}, f\right)_{g}}{\left(\phi_{a, i}^{*}, \phi_{a, i}\right)_{g}} \frac{\left(\phi_{b, j}^{*}, h\right)_{g}}{\left(\phi_{b, j}^{*}, \phi_{b, j}\right)_{g}}\left(\phi_{a, i}, \phi_{b, j}\right)_{g}
$$

Applying now item 3 from Theorem 2.1 as well as item 2 from Lemma 2.1 we obtain

$$
\begin{aligned}
& \text { Type } 00:(f, h)_{g}=\sum_{i, j} \frac{\left(\phi_{0, i}^{*}, f\right)_{g}}{\phi_{z}\left(1 ; z_{0, i}\right)} \frac{\left(\phi_{0, j}^{*}, h\right)_{g}}{\phi_{z}\left(1 ; z_{0, j}\right)} \frac{\phi_{\xi}\left(1 ; z_{0, i}\right) \phi_{\xi}\left(1 ; z_{0, j}\right)}{z_{0, i}+z_{0, j}}, \\
& \text { Type } 01:(f, h)_{g}=\sum_{i, j} \frac{\left(\phi_{0, i}^{*}, f\right)_{g}}{\phi_{z}\left(1 ; z_{0, i}\right)} \frac{\left(\phi_{1, j}^{*}, h\right)_{g}}{\phi_{\xi z}\left(1 ; z_{1, j}\right)} \frac{\phi_{\xi \xi}\left(1 ; z_{0, i}\right) \phi\left(1 ; z_{1, j}\right)}{z_{0, i}+z_{1, j}}, \\
& \text { Type } 12:(f, h)_{g}=\sum_{i, j} \frac{\left(\phi_{1, i}^{*}, f\right)_{g}}{\phi_{\xi z}\left(1 ; z_{1, i}\right)} \frac{\left(\phi_{2, j}^{*}, h\right)_{g}}{\phi_{\xi \xi z}\left(1 ; z_{2, j}\right)} \frac{\phi_{\xi \xi \xi}\left(1 ; z_{1, i}\right) \phi\left(1 ; z_{2, j}\right)}{z_{1, i}+z_{2, j}}, \\
& \text { Type } 22:(f, h)_{g}=\sum_{i, j} \frac{\left(\phi_{2, i}^{*}, f\right)_{g}}{\phi_{\xi \xi z}\left(1 ; z_{2, i}\right)} \frac{\left(\phi_{2, j}^{*}, h\right)_{g}}{\phi_{\xi \xi z}\left(1 ; z_{2, j}\right)} \frac{\phi_{\xi}\left(1 ; z_{2, i}\right) \phi_{\xi}\left(1 ; z_{j, 2}\right)}{z_{2, i}+z_{2, j}} .
\end{aligned}
$$

We now define the weights $b_{j}, a_{j}$ generating the measures $\mathrm{d} \beta=\sum_{j} B_{j} \delta_{z_{j}}, d \alpha=\sum_{j} A_{j} \delta_{z_{j}}$ respectively, as residues of $W \mathrm{~s}$ or $Z \mathrm{~s}$ :

$$
\begin{array}{ll}
\text { Type } 00: A_{i}=\frac{\phi_{\xi}\left(1 ; z_{0, i}\right)}{\phi_{z}\left(1 ; z_{0, i}\right)}, & B_{j}=\frac{\phi_{\xi}\left(1 ; z_{0, j}\right)}{\phi_{z}\left(1 ; z_{0, j}\right)}, \\
\text { Type } 01: A_{i}=\frac{\phi_{\xi \xi}\left(1 ; z_{0, i}\right)}{\phi_{z}\left(1, z_{0, i}\right)}, & B_{j}=-\frac{\phi\left(1 ; z_{1, j}\right)}{\phi_{\xi z}\left(1 ; z_{1, j}\right)}, \\
\text { Type } 12: A_{i}=\frac{\phi_{\xi \xi}\left(1, z_{1, i}\right)}{\phi_{\xi z}\left(1, z_{1, i}\right)}, & B_{j}=-\frac{\phi\left(1, z_{2, j}\right)}{\phi_{\xi \xi z}\left(1, z_{2, j}\right)}, \\
\text { Type } 22: A_{i}=-\frac{\phi_{\xi}\left(1, z_{2, i}\right)}{\phi_{\xi \xi z}\left(1, z_{2, i}\right)}, & B_{j}=-\frac{\phi_{\xi}\left(1, z_{2, j}\right)}{\phi_{\xi \xi z}\left(1, z_{2, j}\right)},
\end{array}
$$

and thus indeed

$$
(f, h)_{g}=\sum_{i, j}\left((-1)^{a} \phi_{a, i}^{*}, f\right)_{g}\left((-1)^{b} \phi_{b, j}^{*}, h\right)_{g} \frac{A_{i} B_{j}}{z_{a, i}+z_{b, j}} .
$$

Thus, in view of Remark 3.1, we obtain

$$
(f, h)_{g}=\sum_{i, j} \hat{f}_{1}\left(z_{a, i}\right) \hat{h}_{b}\left(z_{b, j}\right) \frac{A_{i} B_{j}}{z_{a, i}+z_{2, j}}=\int \frac{\hat{f}_{a}(x) \hat{h}_{b}(y)}{x+y} d \alpha(x) d \beta(y)=\left\langle\hat{f}_{a} \mid \hat{h}_{b}\right\rangle .
$$

Remark 3.3. Expanding an arbitrary $f \in L_{g}^{2}[0,1]$

$$
f=\sum_{i} \frac{\left(\phi_{a, i}^{*}, f\right)_{g}}{\left(\phi_{a, i}^{*}, \phi_{a, i}\right)_{g}} \phi_{a, i}
$$

allows one to conclude that

$$
\delta\left(\xi, \xi^{\prime}\right):=\sum_{i} \frac{\phi_{a, i}(\xi) \phi_{a, i}^{*}\left(\xi^{\prime}\right)}{\left(\phi_{a, i}^{*}, \phi_{a, i}\right)_{g}}
$$

plays the role of the Dirac delta on $L_{g}^{2}[0,1]$. 
It is now elementary to find the inverse Fourier transforms

Lemma 3.1. Consider the BVP of type a. Let $\left\{z_{a, i}\right\}$ be the corresponding spectrum and let $d \nu_{a}=\sum_{i} \delta_{z_{a, i}}$ be an associated measure. Then the inverse generalized Fourier transform of type a is given by

$$
(-1)^{a} \int \hat{f}_{a}(z) \frac{\phi(\xi ; z)}{\left(\phi_{a}^{*}(z), \phi(z)\right)_{g}} d \nu_{a}(z)
$$

Proof. This is a direct computation:

$$
\begin{aligned}
& (-1)^{a} \int \hat{f}_{a}(z) \frac{\phi(\xi ; z)}{\left(\phi_{a}^{*}(z), \phi(z)\right)_{g}} d \nu_{a}(z)=(-1)^{a} \sum_{i} \hat{f}_{a}\left(z_{a, i}\right) \frac{\phi_{a, i}(\xi)}{\left(\phi_{a, i}^{*}, \phi_{a, i}\right)_{g}}=\sum_{i}\left(\phi_{a, i}^{*}, f\right)_{g} \frac{\phi_{a, i}(\xi)}{\left(\phi_{a, i}^{*}, \phi_{a, i}\right)_{g}}= \\
& \int_{0}^{1} \sum_{i} \frac{\phi_{a, i}(\xi)}{\left(\phi_{a, i}^{*}, \phi_{a, i}\right)_{g}} \phi_{a, i}^{*}\left(\xi^{\prime}\right) f\left(\xi^{\prime}\right) g\left(\xi^{\prime}\right) d \xi^{\prime}=\int_{0}^{1} \delta\left(\xi, \xi^{\prime}\right) f\left(\xi^{\prime}\right) g\left(\xi^{\prime}\right) d \xi^{\prime}=f(\xi) .
\end{aligned}
$$

There are in general two measures associated with each type of the BVP, one generated by $W$ the other by $Z$. One can use either one of the them instead of the measure $d \nu$. We give as an example the relevant statement for the the case of the BVP of Type 2, both the degenerate and the nondegenerate one.

Lemma 3.2. The inverse generalized Fourier transform of Type 2 is given by

$$
\int \hat{f}_{2}(z) \frac{\phi(\xi ; z)}{\phi_{\xi}(1 ; z)} d \beta(z)
$$

Proof. From the definition of $d \beta$ given in Theorem 2.3 we see that

$$
\begin{aligned}
& \int \hat{f}_{2}(z) \frac{\phi(\xi ; z)}{\phi_{\xi}(1 ; z)} d \beta(z)=\sum_{i} \hat{f}_{2}\left(z_{2, i}\right) \frac{\phi_{2, i}(\xi)}{\phi_{\xi}\left(1 ; z_{2, i}\right)} b_{i}=\sum_{i} \hat{f}_{2}\left(z_{2, i}\right) \frac{\phi_{2, i}(\xi)}{\phi_{\xi}\left(1 ; z_{2, i}\right)}\left(-\frac{\phi_{\xi}\left(1 ; z_{2, i}\right)}{\phi_{\xi \xi z}\left(1, z_{2, i}\right)}\right)= \\
& \sum_{i}\left(\phi_{2, i}^{*}, f\right)_{g} \frac{\phi_{2, i}(\xi)}{\phi_{\xi}\left(1 ; z_{2, i}\right)}\left(-\frac{\phi_{\xi}\left(1 ; z_{2, i}\right)}{\phi_{\xi \xi z}\left(1 ; z_{2, i}\right)}=\sum_{i}\left(\phi_{2, i}^{*}, f\right)_{g} \frac{\phi_{2, i}(\xi)}{\left(\phi_{2, i}^{*}, \phi_{2, i}\right)_{g}},\right.
\end{aligned}
$$

where in the last two steps we used Remark 3.1 and equation (2-23) respectively. Thus

$$
\int \hat{f}(z) \frac{\phi(\xi ; z)}{\phi_{\xi}(1 ; z)} d \beta(z)=\int_{0}^{1} \sum_{i} \frac{\phi_{2, i}(\xi) \phi_{2, i}^{*}\left(\xi^{\prime}\right)}{\left(\phi_{2, i}^{*}, \phi_{2, i}\right)_{g}} f\left(\xi^{\prime}\right) g\left(\xi^{\prime}\right) d \xi^{\prime}=\int_{0}^{1} \delta\left(\xi, \xi^{\prime}\right) f\left(\xi^{\prime}\right) g\left(\xi^{\prime}\right) d \xi^{\prime}=f(\xi)
$$

The generalized Fourier transform can be used to give the following interpretation of the Cauchy biorthogonal polynomials associated to discrete, finite positive measures $d \alpha, d \beta$. 
Theorem 3.2. Biorthogonal polynomials Let us consider the sequence $\chi_{j}:=\chi_{\left(\xi_{n-j}-\epsilon, \xi_{n-j}+\epsilon\right)}$ of indicator functions enclosing points $\xi_{n-j}$ of the support of the measure $g$ appearing in the cubic string equation (2-1), with $\epsilon$ small enough to ensure non overlapping supports. Furthermore, let us consider the generalized Fourier transforms for Types $a, b$ as in Theorem 3.1 and define:

$$
p_{j}(x):=\hat{\chi}_{a, j}(x), \quad q_{j}(y):=\hat{\chi}_{b, j}(y) .
$$

Then, $\left\{p_{j}, q_{k}\right\}$ are (un-normalized) Cauchy biorthogonal polynomials, that $i s<\hat{\chi}_{a, i} \mid \hat{\chi}_{b, j}>=0, i \neq j$ and $<\hat{\chi}_{a, i} \mid \hat{\chi}_{b, i}>\neq 0$.

Proof. Both $\hat{\chi}_{a, i}(x)$ and $\hat{\chi}_{b, j}(y)$ are polynomials in $x, y$ respectively, whose degrees are $\operatorname{deg} \hat{\chi}_{a, j}(x)=$ $\operatorname{deg} \hat{\chi}_{b, j}(y)=j$ by (2-5). The biorthogonality follows from Theorem 3.1.

\section{Acknowledgments}

We would like to to thank R. Beals for sharing with us his insight regarding the Parseval Identity for an ordinary inhomogeneous string of M.G. Krein which influenced the formulation of Theorem 3.1.

\section{References}

[1] M. G. Krein. On inverse problems for a nonhomogeneous cord. Doklady Akad. Nauk. SSSR (N.S), 82:669-672, 1952 .

[2] M. G. Krein. On a generalization of investigations of stieltjes. Doklady Akad. Nauk. SSSR (N.S), $87: 881-884,1952$.

[3] A. Degasperis and M. Procesi. Asymptotic integrability. In A. Degasperis and G. Gaeta, editors, Symmetry and perturbation theory (Rome, 1998), pages 23-37. World Scientific Publishing, River Edge, NJ, 1999.

[4] A. Degasperis, D. D. Holm, and A. N. W. Hone. A new integrable equation with peakon solutions. Theoretical and Mathematical Physics, 133:1461-1472, 2002. Preprint nlin.SI/0205023.

[5] H. Lundmark and J. Szmigielski. Degasperis-Procesi peakons and the discrete cubic string. IMRP Int. Math. Res. Pap., (2):53-116, 2005.

[6] M. Bertola, M. I. Gekhtman, and J. Szmigielski. Peakons and Cauchy Biorthogonal Polynomials. arXiv:0711.4082, 2007.

[7] M Bertola, M Gekhtman, and J Szmigielski. The Cauchy two-matrix model. Comm. Math. Phys., 287(3):983-1014, 2009.

[8] I. S. Kac and M. G. Krein. On the spectral functions of the string. Amer. Math. Soc. Transl., 103(2):19-102, 1974. 